\title{
Hortobágy National Park
}

\section{- An Island of Undisturbed Nighttime Environment}

\author{
István Gyarmathy ${ }^{1,2, *}$, Zoltán Kolláth² \\ ${ }^{1}$ Hortobágy National Park, Debrecen, H-4024, Hungary \\ ${ }^{2}$ Eötvös Loránd University, Savaria Department of Physics, Szombathely, H-9700, Hungary
}

Received 12 December 2016, Accepted 17 May 2017

\begin{abstract}
National parks and protected areas have an important role in protecting starry sky and the undisturbed nighttime environment. Hortobágy which is one of the darkest areas in Hungary, became an International Dark Sky Parks recently. Its significance is mostly related to the protection of the high biodiversity which is endangered by the effects of light pollution. A special monitoring program has been started to survey the nocturnal species and also to monitor the quality of the night sky using digital cameras. Stargazing night walks are frequently organized. There is a high interest by the general public to attend these night adventures.
\end{abstract}

Keywords: light pollution and national parks, dark-sky parks, light pollution monitoring, astro-tourism

\section{Introduction of Hortobágy National Park}

The Hortobágy National Park is situated on the former floodplain of the Tisza river (the park's area is 82000 hectares, the nearest city is Debrecen, 35 kms East from the park's border, see Figzre 7. too).The park is the first proclaimed and biggest Hungarian national park, also a World Heritage site, Ramsar site, Natura 2000 site and UNESCO Biosphere reserve.

Hortobágy is an almost flat plain landscape, great and continuous grassland area with wetland mosaics, the most extended in its category in Europe. It is occupied by alkaline marshes, meadows, dry alkaline pastures and remnant loess-steppe vegetation [1].

Hortobágy is most famous for its rich avi-fauna. The number of nesting species is 159 and there are additional 178 species which are regular or irregular visitors.

Hortobágy is generally the best birding place in Hungary (possibly in the whole Karpathian Basin) and the most important IBA (Important Bird Area) too. Migration is particularly significant (Fig. 3.).

The masses of migration are remarkable too: for instance 100,000-300,000 of grey geese (Anser sp.), appr. 100,000 cranes (Grus grus), or 50,000-200,000 Ruffs stop at Hortobágy (the last one basically while shallow-water covered conditions occur in grasslands). 10-20 years ago hundreds of thousands of different duck stopped here too, but their mass has been less significant recently. 


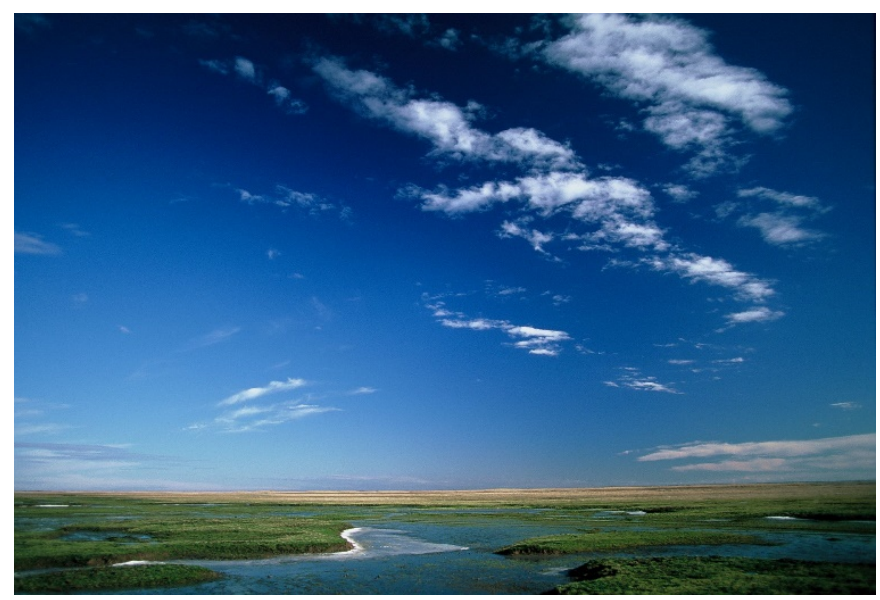

Fig. 1. The first slogan of the park expresses the main feature of the landscape: the endless flat grassland area is like the sea: Hortobágy, where the Earth and Sky meet (photo: Gábor Kovács)

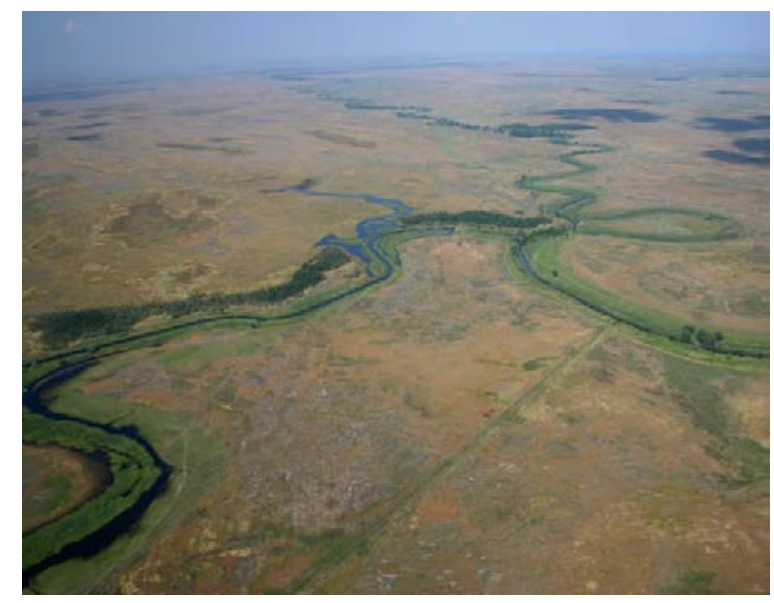

Fig. 2. Hortobágy from above. You can see the Hortobágy river and the typical steppe with wetland mosaics (photo: Szilvia Gőri)

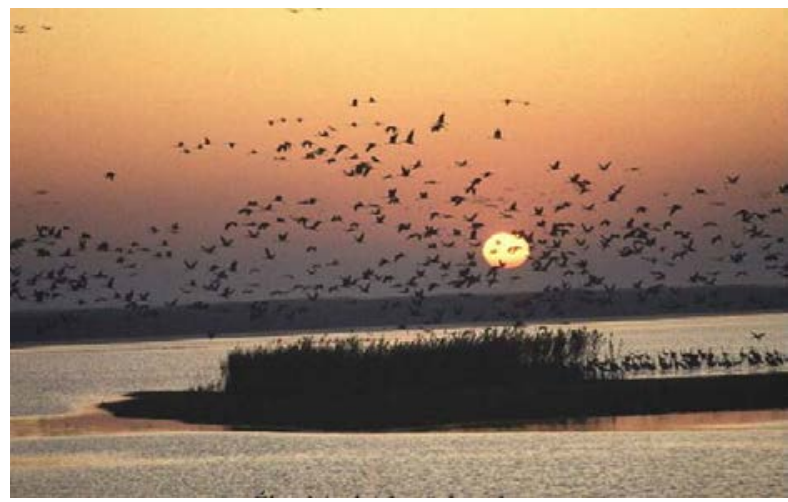

Fig. 3. The obtrusive light-free environment is essential for the wildlife as e.g. insects at night and the protection of migratory birds [2] (photo: Attila Szilágyi) 


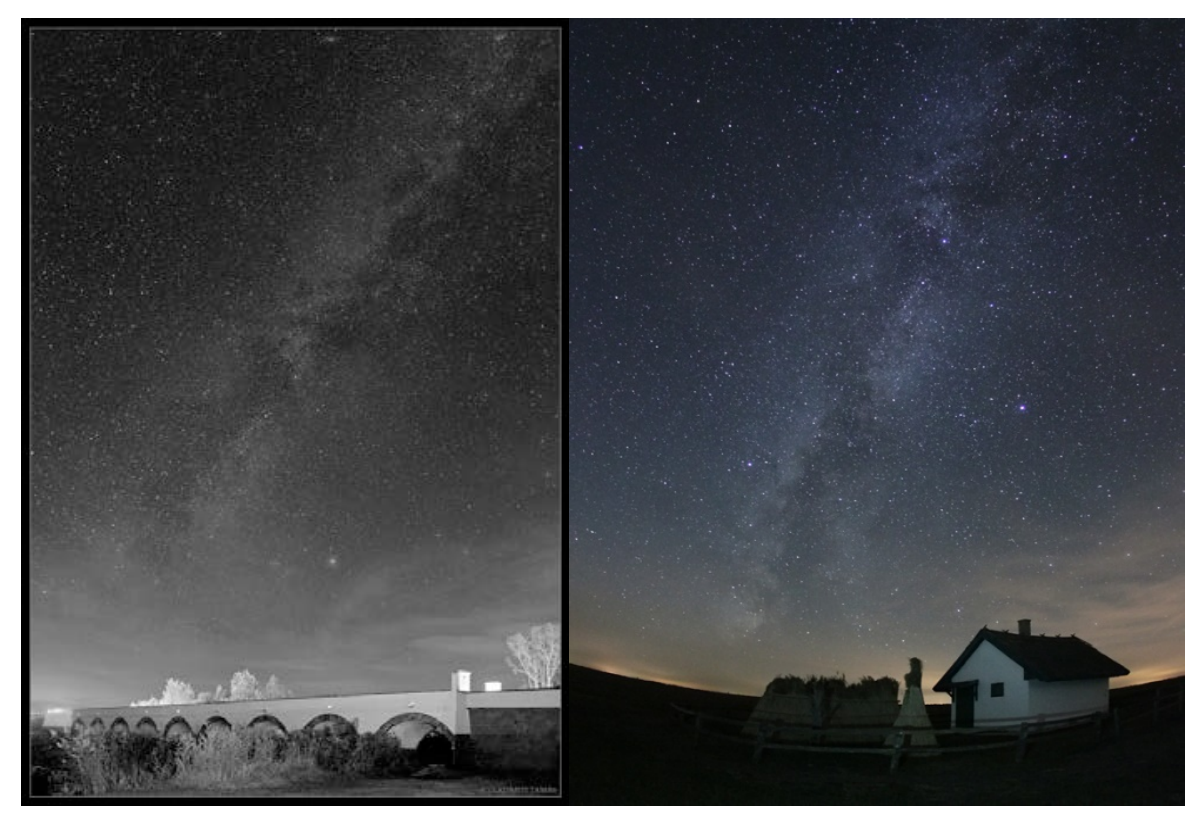

Fig. 4. The „Nine-hole bridge” is one of the symbols of the WH site, with the Milky Way can be the symbol of the Dark-sky park (left) and a traditional shepherd building with the Milky way (right) photo: Tamás Ladányi.

Hortobágy got its World Heritage (WH) Diploma in the cultural category - as it is related to traditional land use and ancient shepherd culture, however Hortobágy's biodiversity -as it was mentioned above - is also very high and the park's significance is very important in preserving nature (Figure 4).

Hortobágy is one of the last guardians of living shepherd tradition. Shepherd culture has deep interrelation and interdependence with the natural environment. Pastures of Hortobágy are scattered with traditional buildings of ancient pastoral activity. There are many evidences in ethnographical works of the starry-sky knowledge of the shepherds (Figure 4, right panel).

„... Regarding the Great Plains in today's Hungary, the knowledge of stars of Hortobágy people, folk traditions associated with the stars is richer, more colorful than anywhere else in Hungary ...” (Győző Zsigmond ethnographer) [3]

„The shepherds knew the hours from the procession of stars. The Big Dipper, the Pleiades, the Kaszáscsillag (Sirius) and the Milky Way all turn and indicate where we are in the night.”

(local ethnographic collection)

Sándor Szücs famous ethnographer writes in the Puszta Chronicle that „they only informed about the world in „pastoral style” observing the stars and the ground and grasses.” [4]

As we can see from the citations above, shepherds had very good knowledge of the stars, the phenomena related to the sky. Their mythology and also their daily life has got many connections with the starry sky. So the protection of dark sky is important in preserving folk traditions too.

Shepherd culture was an important part of the World Heritage nomination. Now Hortobágy according to our opinion should be part of UNESCO Astronomy and World Heritage program too, as an archeo-astronomy or ethnoastronomy site (see Figure 5). 


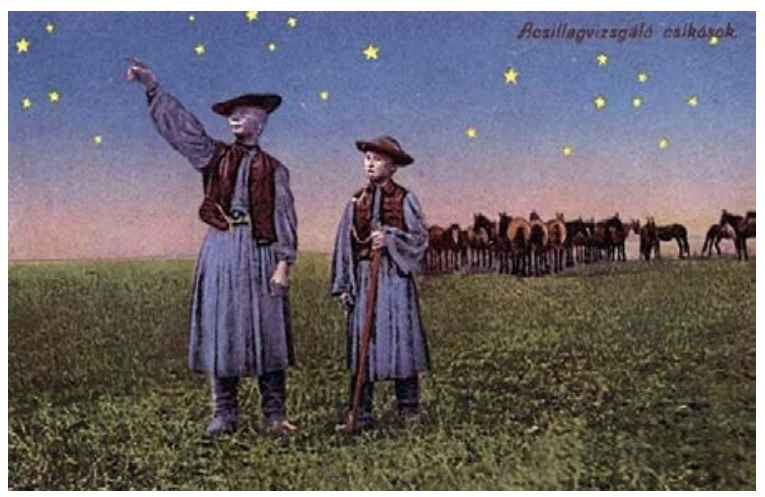

Fig. 5. Star-gazing horse-herds - old postcard from the early 1900s (image: Photo archive of HNP)

\section{Hortobágy as a dark sky park}

Hortobágy is one of the biggest unpopulated and darkest areas in Hungary. Hortobágy got its international dark sky park (or Starry Sky Park - SSP) diploma from the International Dark Sky Association in 2011 according to the nomination submitted in 2010 (Fig.6.).

The starry sky park's significance is mostly related to undisturbed nighttime landscape, the shepherd tradition related to starry sky and the protection of the high biodiversity, especially the great number of migrating bird species and special nocturnal insect species. It is notable that many of the important breeding and nesting bird species (geese, cranes, spoonbills, etc.) and other species, especially many rare insect species are light pollution sensitive.

To be a starry sky park is an important tool to protect the nocturnal wildlife habitats as well as the landscape values of Hortobágy [5] as an outstanding and an unaltered wilderness area in the Great Hungarian Plain, in the middle of Europe. Now the dark-sky values also provide new attraction to the area: the park became also an astrotourism destination.

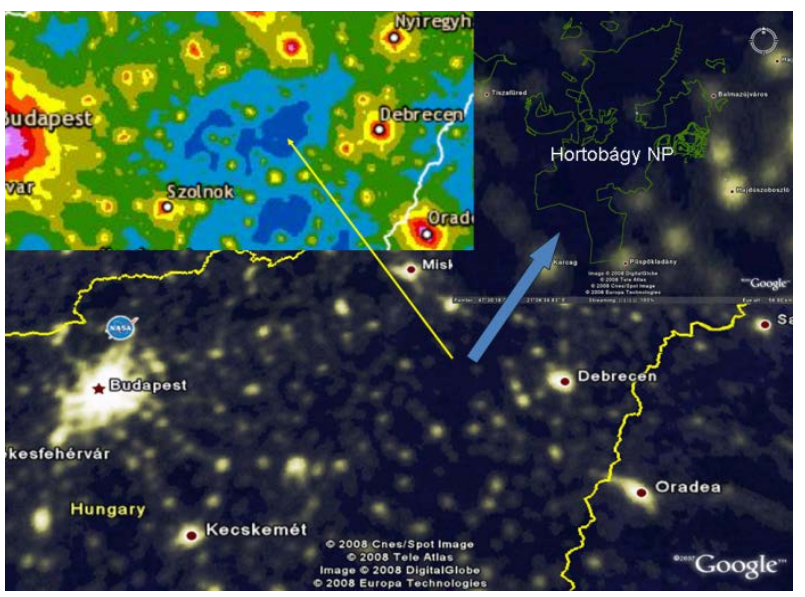

Fig. 6. Hortobágy National Park on the Google Earth nighttime satellite map and on the new World Atlas of artificial sky brightness [6] Both maps show: the area is as intact and undisturbed nighttime as daytime. The lack of light-pollution can be one of the measures of naturalness!

Astro-tourist is traveling for the purpose of astronomy or only enjoys the beauty of the pristine starry sky. Starry sky parks can be main places for developing astro-tourism and amateur astronomy. 
These darkest corners of the planet where the environment is not disturbed at night by artificial light, have not only ecological functions, but also educational and touristic values.

Hortobágy Starry Sky Park organizes nighttime walks, special interpretive programs related to the dark-sky values and good lighting practices. There is a high interest by the general public to attend these night adventures. The Park has different programs designed to show the values of the park (Fig.7.), and is planning a new visitor center with exhibition dedicated to the dark sky park).

Astronomy became part of the park's Field Study Center's curriculum. The park has recently established a public astronomical observatory as part of the development of the Center (Fig.8). It is equipped also by all-sky camera and permanent SQM too.

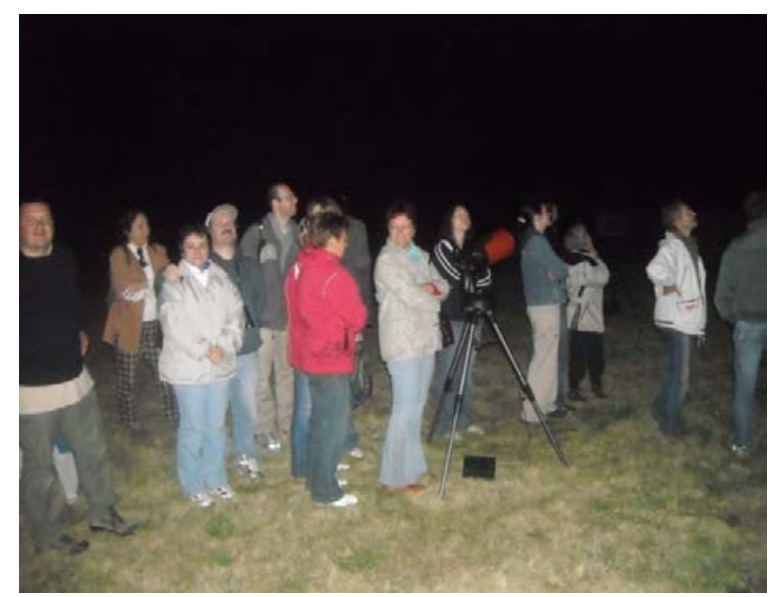

Fig. 7. Group of astro-tourists in the park (photo: István Gyarmathy)

\section{Lighting regulations}

According to the Park's regulations the following conditions should be satisfied by any outdoor lighting in Hortobágy [7]:

- Only fully shielded fixtures can be used, and they should be installed and serviced so that no light should be emitted above the horizontal plane.

- Color temperature cannot exceed $3000 \mathrm{~K}$.

- The maximum allowable light output (luminous flux) per fixture is 1800 lumens.

If the total luminous flux of a premise or realty exceeds 10000 lumens, a detailed lighting permit plan should be prepared.

- Any construction should be started only after approval by Hortobagy National Park Directorate as the competent nature conservation management organization, and by IDA Hungary.

- The illumination levels cannot exceed the minimum norms available for the given purpose.

- Outdoor lights can only be used when pedestrian or considerable vehicular traffic is expected. Motion sensors or time switches are preferred in locations with infrequent traffic.

- Use the most energy efficient lamps are preferred.

The Park included the articles of the Lighting Plan of the Starry Sky Park to its Management Plan to protect the natural and scenic values of the undisturbed nighttime environment. The lighting regulation and zoning policy provide a possibility to control artificial lights within the park.

The park has cooperation agreements with local and national conservation and astronomy NGOs to protect the area’s dark sky values as well as with the local stakeholders. 


\section{Sky Quality at the Hortobágy National Park}

At Hortobágy we have used the same methods as in the case of the Zselic Landscape Protection Area. [8]

In general, it can be concluded, that inside and in the close neighborhood of the Hortobagy National Park there are no light sources which contribute to the luminance of the night sky. Then a few measurements at the perimeter can be sufficient to describe the quality of the night sky. However, a more thorough project has been started, since the markings of Hortobágy SSP (almost perfectly plain territory, good view along the horizon) provide good opportunity to scientific studies based on the observed data.

Our SQM measurements show that the quality of the night sky is everywhere in the National Park is better than $21.0 \mathrm{mag} / \mathrm{arcsec} 2$ (we use here mag/arcsec2 as standard unit). The typical values are around 21.3-21.4 , in ideal weather conditions, it can reach 21.5 - 21.,6 (Figure 8). All of our measurements were taken with the device pointing to the zenith. The necessary conditions to perform a field survey were the followings:

- Moonless night.

- No clouds or fog.

- The Sun is at least 18 degrees below the horizon (astronomical twilight).

- No direct light from artificial sources reaches the detector of the device.

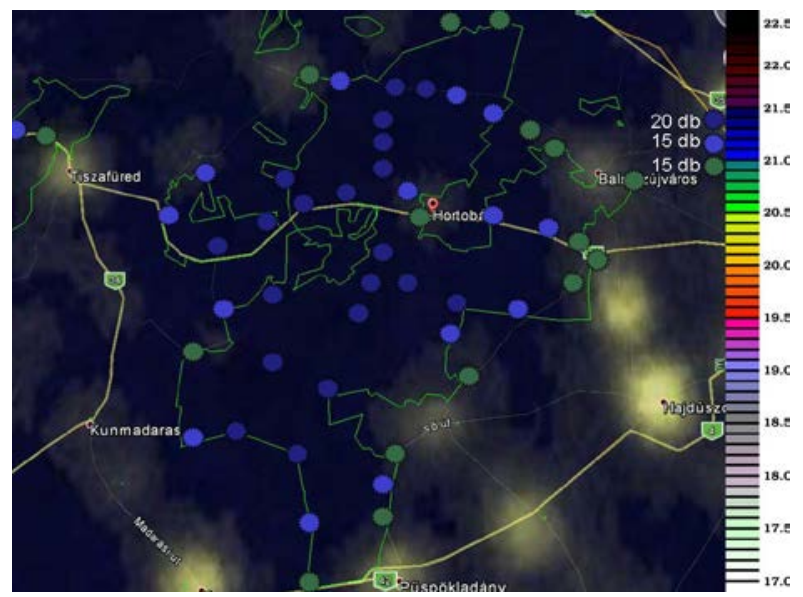

Fig. 8. SQM measurements map of Hortobágy based on our survey (image: Google Earth Nighttime Satellite Map with our measurement results)

We prove the quality of Hortobágy's sky quality using permanent SQM, the data can be reached on the internet (Figure 9) [9].

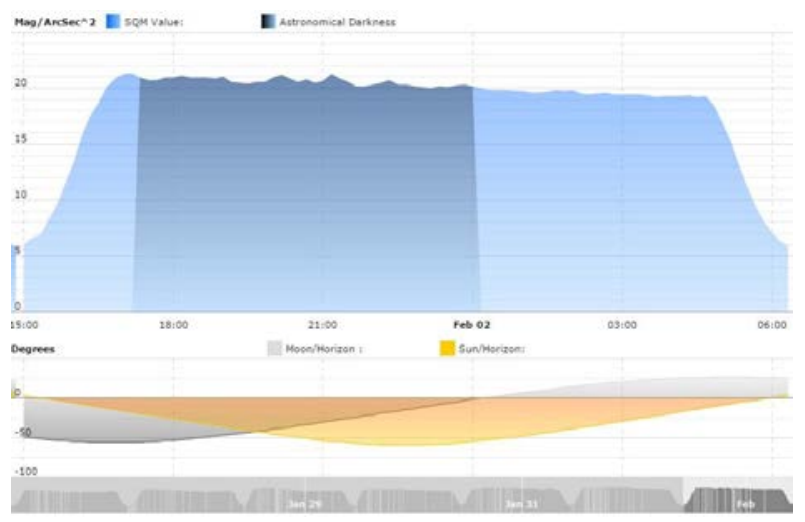

Fig. 9. Permanent SQM data on SQM Network (image: SQM Network) 
Local settlements have only a minimal effect on the quality of the sky. The luminance is only slightly increased in the vicinity of small villages. The lights from the cities of Hajdúszoboszló (population 23000, 11 kms SouthEast from the park's border), Balmazújváros (population 18000, at the park's NE border), Karcag (population 20000, 8 kms from the park’s SW border), Nádudvar (population 9200, at the park’s Southern border), Tiszafüred (population 11200, $7 \mathrm{kms}$ from the Western border), Nagyhegyes (population 2700, at the park's Eastern border) and Polgár (population 8400, 6 kms NW from the park’s border) are the main sources of light pollution. However these cities do not have any significant effect on the SQM values, i.e. the quality of the night around the zenith inside the park (see also Figure 12).

Based on fisheye all sky DSLR images the luminance distribution on the night sky is determined.

(Our standard settings for light pollution monitoring in dark locations are the followings:

- Canon EOS 50D camera (some parallel tests with EOS 350D, 450D and 300D)

- Sigma 4.5mm f2.8 EX DC circular fisheye lens

- ISO 800 setting

- $\mathrm{T}=180 \mathrm{~s}$ exposure time

- $\mathrm{f}=2.8-3.5$ aperture

The processing of DSLR images is described in Kolláth \& Dömyén, this volume.)

These images show that the effect of nearby settlements is significant only close to the horizon. The deterioration of the nocturnal sky for zenith distances less than 80 degrees is acceptable. This fact is proved by the total light flux of the sky more than 20 degrees above the horizon.

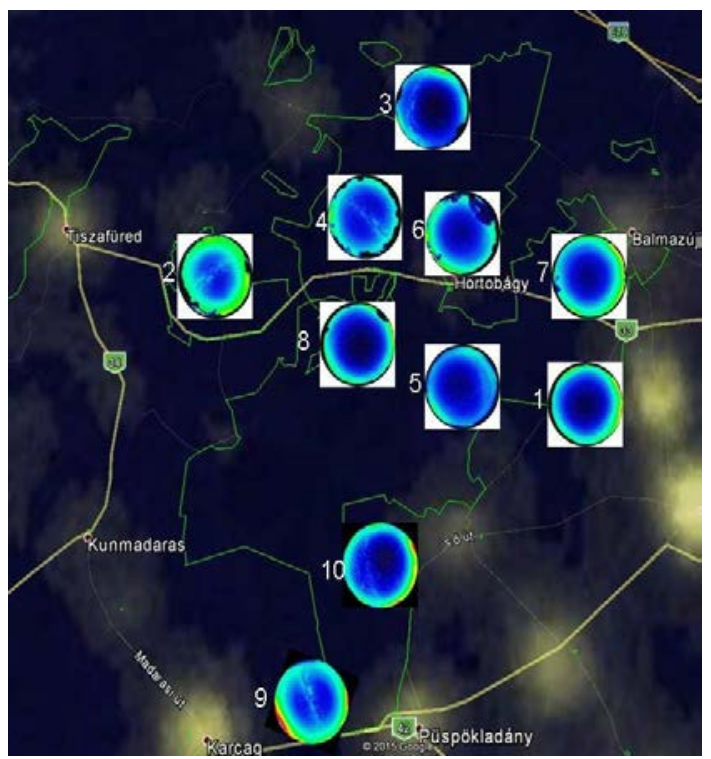

Fig. 10. Hortobágy’s light pollution map: our processed DSLR fish-eye lens photos on Google-Earth nighttime satellite map

The luminance calibrated images are displayed on a false color scale to display the luminance distribution on the sky. The major obtrusive sources can be clearly identified when such images (after a mirroring) are displayed on a map of the region (Figure 10). It is also possible to get the spatial dependence of sky quality inside the park. 


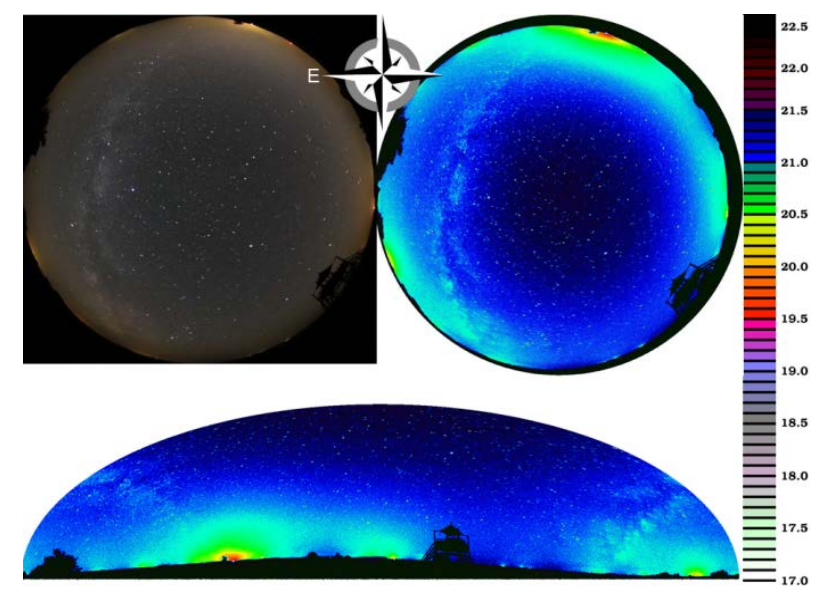

Fig. 11. Light pollution at the Northern,,Cserepes” site (No 4 on Fig. 10.) The biggest light dome is of Polgár

The full sky luminance maps give the opportunity to determine the sky brightness as a function of zenith distance. For the derivation of this function, we selected concentric rings at different zenith angles of a width of 2 degrees. For each rings the maximum, the minimum and the mean value of the radiance is calculated. On the right panel of Figure 12, we present all these three curves. This method provides a compact summary on the luminance distribution in the sky. The local maximum in brightness in the maximum curve is due to the presence of the Milky Way.

Such profiles of sky quality at different azimuths provide quantitative data on the light pollution from different sources. According to our experience, at zenith distances more than 70-75 degrees the measurements strongly depend on the neighborhood, as plants and buildings obscure the view. A useful quantity is the average luminance in a narrow range of zenith distance (e.g. 0-30 or 30-60 degrees). At low light pollution locations the nearby sources have only limited effect on the sky at less than 40 degrees below the zenith. As seem from Figure 12, the mean value drops below $21 \mathrm{mag} /$ arsce 2 only below 50 degrees zenith angle at the specific location.
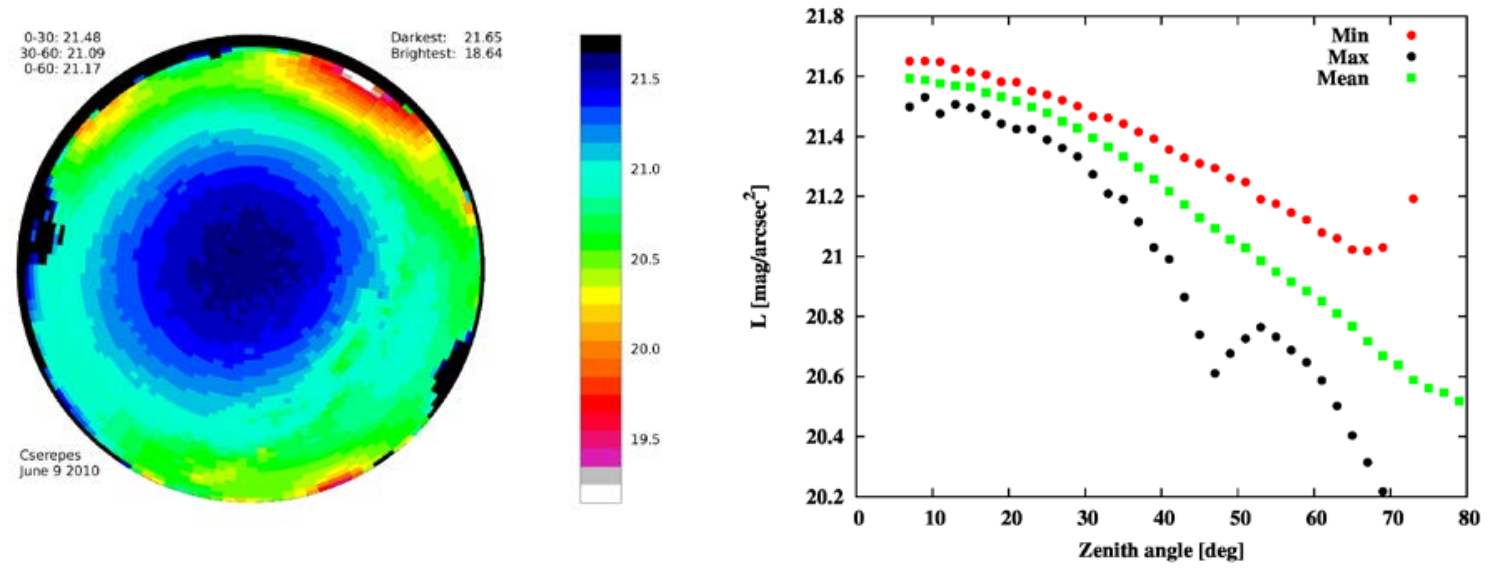

Fig. 12. Full sky luminance map at Cserepes location 
That is clear, that all anthropogenic light sources within hundreds of kms should be taken into consideration to fit perfectly the nigh sky luminance. Light domes of cities at larger distances cannot be identified on the images, but they can contribute to the general increase of sky luminance.

The best solution would be a nationwide law against light pollution, which has been already initiated.

All the measurements prove that the quality of the sky at the Hortobagy National Park is better than the minimal limits for "silver tier” (according to International Dark Sky Association’s terminology and requirements), and this state can be conserved for further generations.

\section{Conclusion}

The conservationist community has growing interest in light pollution and its ecological effects worldwide.

The Hungarian protected area network (national parks, wildlife reserves) almost overlaps with the dark sky areas. This fact supports their mission in protecting dark skies.

They also provide possibility for light pollution monitoring with both physical and biological methods. Hortobágy Starry Sky Park leads light pollution monitoring using DSLR camera with fisheye-lens across its area and a permanent SQM has been installed. A biomonitoring program on the effects on nocturnal species, especially insects is under construction. The sky brightness measurements prove the good quality of the undisturbed night sky. These results strengthen the commitment of the park administration to continue the program.

Hortobágy SSP is also a good destination for astro-tourism which is a new and developing type of eco-tourism.

\section{References}

[1] Tardy, J. (1999). Conserving Hungary’s Heritage. Természetbúvár Kiadó, Budapest.

[2] Rich, C. \& Longcore, T. (2006). Ecological Consequences of Artifical Night Lighting, Island Press, Washington.

[3] Zsigmond, Gy. (2005). Az erdélyi magyarság csillagnévhasználatáról. Available from: http://www.csillagaszat.hu/csilltort/magyar-csillagaszattortenet/magyar-nepi-csillagnevek/az-erdelyimagyarsag-csillagnevhasznalatarol/.

[4] Szúcs, S. (1946). Pusztai krónika, Misztótfalusi Kiadó.

[5] Kolláth, Z. \& Gyarmathy, I. (2009). Dark Sky Program in Hungary. Dark Skies Awareness homepage: http://www.darkskiesawareness.org/dspp-h.php.

[6] Falchi, F. et al. (2016). World Atlas of artificial sky brightness. Available from: http://cires.colorado.edu/Artificiallight

[7] Lighting Plan of the Hortobágy Starry Sky Park.

[8] Kolláth, Z. (2010) Journal of Physics: Conference Series 218012001.

[9] SQM Network homepage: http://www.sqmnetwork.com. 